\title{
Providing Global Management Functions for ATM Networks
}

\author{
Bernhard Thurm ${ }^{1}$ and Hajo R. Wiltfang ${ }^{2}$ \\ ${ }^{1}$ Institute of Telematics, University of Karlsruhe \\ D-76128 Karlsruhe, Germany \\ thurmetelematik.informatik.uni-karlsruhe.de \\ 2 Deutsche Telekom AG, IP Backbone Engineering, D-48014 Muenster, Germany \\ Hajo.Wiltfangetelekom.de
}

\begin{abstract}
Modern computer networks with their increasing bandwidth and their ability of QoS support require adequate management solutions. For ATM networks, a management system especially needs global functions combining the local management functionality distributed over all individual network devices to network-wide functions in order to manage e.g. end-to-end connections and their QoS aspects. In this context, the following paper presents an approach to ATM network management which introduces a function-oriented architecture. Based on this architecture, the functionality of two developed management entities, the ATM Network Monitor and the PVC Manager, which are both designed for important network-wide management functions like topology discovery, connection management, and QoS monitoring are described in detail.

Keywords: Distributed management, Network-wide management functions, ATM

networks, ATM management architecture and applications
\end{abstract}

\section{Introduction}

Computer-based communication has become a more and more central aspect for modern society. Besides traditional data communication such as email and file transfer, forthcoming applications like multimedia and tele-conferencing increase the need for integrated networks supporting Quality-of-Service (QoS) on a per connection basis. An important example in this area is the ATM (Asynchronous Transfer Mode) technology which provides virtual connections (VCs) based on asynchronous cells and a dedicated QoS associated with each connection.

For integrated networks such as ATM, the number and quality of additionally offered services comes along with a rising complexity of the network technology in general and of the required network devices in detail. This rising complexity leads to significantly increased requirements on an adequate network management. Especially the QoS aspect together with the connection-oriented communication scheme demands for totally new management functions. In traditional networks, management functions are mainly concentrated on individual network devices, e.g. configuration or monitoring of a router. In case of ATM, new network-wide management functions are needed in order to combine the local functions distributed over all individual network devices with the aim of controlling and monitoring the global functionality of this integrated network 
technology. For example, the management driven set-up of a $\mathrm{VC}$ between two given end points throughout the network requires management functions that are related to more than one individual network device. Existing approaches to ATM management do either not focus on network-wide functions or they rely on special kinds of interfaces to the management of individual devices. For instance, the VIVID system from Newbridge [6] uses the proprietary CPSS (Control Packet Switching System) protocol on a dedicated management VC for providing network-wide functions whereas the xbind architecture [4] is built on top of CORBA [3] which requires a CORBA interface at each device.

To overcome the described demand for network-wide functions, this paper presents a management approach which is based on standardized and widely deployed management interfaces and information as well as a developed function-oriented management architecture. Because of its orientation towards the required functions this architecture is specially designed for supporting network-wide ATM management. All details and characteristics of the so-called FuMA architecture (Function-oriented Management architecture for ATM networks) are described in Sect. 2] Based on FuMA, our work concentrates on the development of network-wide functions for some key areas of ATM network management: topology discovery, VC management, and QoS monitoring. As one main result of our development and implementation efforts, Sect. 3 presents the ATM Network Monitor (ANEMON), a management entity providing functions for all areas mentioned above. In a next step, Sect. 4 describes the developed management application PVC Manager which offers an easy-to-use Web-based interface for all management functions developed so far. In order to show the practical usability of our management functions, Sect. 5 presents the results of some simulations done in a very flexible and scalable simulation environment especially designed for ATM network management simulations. Finally, Sect. 6 summarizes the key points of this paper and gives some ideas for future work.

\section{Function-Oriented Management Architecture for ATM Networks}

The Function-oriented Management architecture for ATM networks (FuMA) [12] has been developed as management framework in order to provide three abstraction levels for ATM management functions. Each of the three levels focuses on different types of management functions, starting with the simple functions distributed over the devices, continuing with the more complex network-wide functions, and ending up with user interface functions. In the following, the three levels element management, management middleware, and management applications will be described in detail (see Fig. 1 ).

At the base level, the element management deals with the management of individual ATM devices. Hiding specific characteristics, the element management offers a welldefined management interface for each ATM device. Our specification of this interface includes the type of management protocol as well as the information provided. In detail, the commonly accepted Simple Network Management Protocol (SNMP) is used for all management communication within FuMA and the management information assumed for each ATM device is based on the standardized ATM-MIB [8]. Moreover, integration 


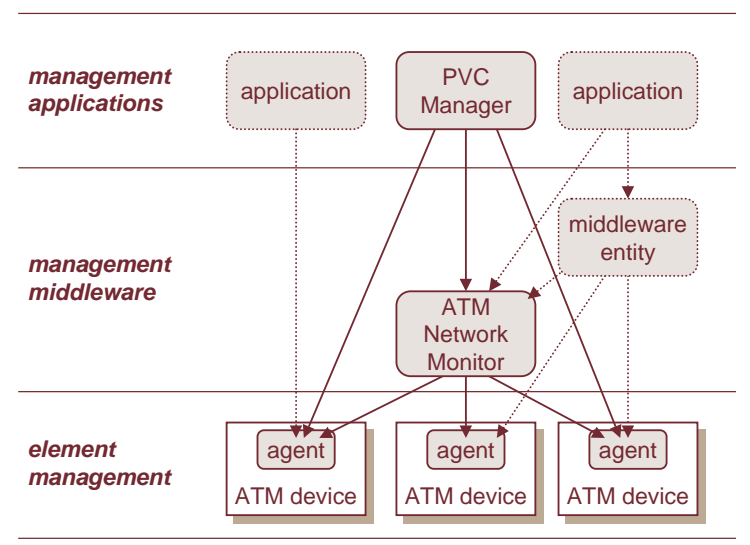

Fig. 1. FuMA Architecture

of devices which do not support the (whole) ATM-MIB can be achieved by using a special kind of proxy agent [7].

At the second level, the management middleware provides network-wide functions which are related to a whole network and thus, to more than only one ATM device. These functions combine the local functions distributed over the individual devices and therefore, are far more complex than the simple element management. The main advantage of the middleware results from the concentration of complex network-wide functions in some modular entities which can be used by multiple other management entities in parallel. Thus, for different management applications, there is no need to implement the middleware functionality by themselves. For example, the ATM Network Monitor ANEMON (see Fig. 1) offers network-wide management functions for topology discovery, VC management, and QoS monitoring, as detailed in Sect. 3 ,

At the third level of FuMA, management applications are located. They typically provide a (graphical) user interface for all functions offered by middleware and element management. Thus, applications directly interact with human users wanting to manage ATM networks. As one example, Fig. 11shows the developed application PVC Manager which will be presented in Sect.4

\section{ATM Network Monitor}

The management middleware entity ATM Network Monitor (ANEMON) has been designed in order to provide network-wide management functionality for the tasks topology discovery, VC management, and QoS monitoring in ATM networks. Based on the modular concept of FuMA middleware, each of these functions can be seen as an independent functional block with its own dedicated MIB describing the implemented functionality. The ANEMON combines three functional blocks within one middleware entity, but the modular structure still remains as shown by Fig. 2 


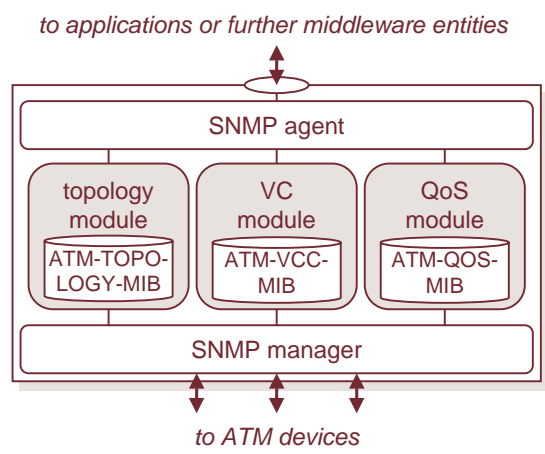

Fig. 2. Architecture of the ATM Network Monitor (ANEMON)

Besides the three functional modules, which will be described in the following sections, the architecture of the ANEMON comprises two further modules (see Fig. 2). The SNMP agent handles the SNMP-based communication with management applications or other middleware entities (see Fig. 1) in order to provide the implemented management functionality of the ANEMON. The amount of management information offered by this agent is given by the superset of all functional module MIBs which will be introduced in the following sections. At the interface to the ATM devices, the SNMP manager of the ANEMON performs the communication needed for obtaining all required information from the devices.

\subsection{Module for Topology Discovery}

Topology information has a very fundamental character for network-wide management functions on which this paper concentrates. In order to identify the ATM devices involved in a network-wide function the performing management entity essentially needs information on the underlying network topology, i.e. which devices are connected to each other. Therefore, the first and most fundamental functionality developed for the ANEMON was the topology discovery which is located within the topology module (see Fig. 2). The management information of this module is specified by the developed ATM-TOPOLOGY-MIB [12 13] which includes information for configuring and controlling the topology discovery as well as providing the resulting topology data.

The algorithm implemented for topology discovery is based on a passive concept, i.e. topology information already distributed between ATM devices is collected and combined to the overall topology. In detail, neighborhood information contained in the atmInterfaceConfTable of the ATM-MIB [8] is used by the algorithm. Starting at one given point, the algorithm computes the overall topology step-by-step by continuously evaluating the distributed neighborhood information. In each step, all neighbors are determined for the evaluated ATM device and then, the search is applied to each neighbor in a depth-first search manner. The developed discovery algorithm terminates at each detected end system or if the configured maximum search depth is reached. More details on this algorithm can be found in [12,13]. 


\subsection{Module for VC Management}

For the management of virtual connections (VCs), two types of network-wide functions can be distinguished, tracing of existing VCs (for any kind of VC) and management (i.e. set-up and disconnect) of permanent VCs (PVCs). For both areas, our VC module (see Fig. (2) provides the basic set of required management functions. Again, the management information for this module is specified by a developed MIB, our ATM-VCC-MIB [9 12 ].

The developed algorithm for VC tracing is very simple because it can take advantage of the topology information already provided by the topology module (see Sect. 3.1 . Based on this information and a given VC starting point, the algorithm computes the trace by continuously evaluating VC switching information on the next ATM device in the topology [12]. The result mainly consists of a set of connection links (VCLs, virtual channel links) which are stored in a special data table of the ATM-VCC-MIB.

The functions developed for PVC management are also based on our topology information, however, they are much more complex. Primarily, this is caused by the fundamental concept for virtual ATM connections which requires a virtual channel connection (VCC) to consist of a series of virtual channel links (VCLs). Each VCL is identified by a combination of VPI (Virtual Path Identifier) and VCI (Virtual Channel Identifier) unique for the underlying physical link. The concatenation of these virtual links is obtained by cross-connecting them at the intermediate ATM devices. Consequently, the management of a whole VCC can only be achieved by considering all distributed devices. As an example, we will illustrate the different steps and algorithms involved in the set-up process of a permanent VCC.

First of all, an 'appropriate' path between two end points has to be calculated. Considering certain constraints (unambiguousness, absence of cycles, optimality) together with the similarity of ATM networks and undirected distance graphs, we developed a solution based on Dijkstras algorithm for the single source shortest paths problem [2]. Necessary modifications primarily were related to the different types of ATM devices (end systems perform no switching) and parallel physical links [9]12].

Step two concerns the selection of a VPI/VCI combination to identify a connection. Locally, the only management information available from the ILMI-MIB and the ATM-MIB [1,8] define certain intervals for the identifiers of virtual channel connections. However, searching through a column of the atmVclTable containing the VPI and VCI values in use can slow down the establishment process if lots of virtual links are configured throughout the network since it has to be performed for each device on the path. Globally, i.e. considering the whole end-to-end span, it is desirable to identify single virtual links of a connection consistently. Therefore, the best trade-off between an arbitrary and a time-consuming full uniform identification is to find a pair of VPIs and VCIs which is usable for the two end points of a connection while for the inner links changes are made only when necessary [9].

In a third step, all virtual links, cross-connects, and traffic parameters have to be created which can be achieved by accessing the atmVclTable, the atmVcCrossConnectTable, and the atmTrafficDescrParamTable of the ATM-MIB located on each device. 


\subsection{Module for QoS Monitoring}

Finally, the QoS module of the ANEMON is designed to provide functions for monitoring QoS parameters in ATM networks. So far, two functional areas are addressed by our QoS module, the monitoring of statistics for physical links within the topology (aggregated link-based QoS) and QoS monitoring for selected VCs which focuses on each VCL of a monitored VC (detailed VCL-based QoS). The management information for both functional groups is specified by our developed ATM-QOS-MIB [[10,12].

The monitoring of physical links is based on the interface information of the MIB-II [5] (e.g., the octets sent and received at each ATM interface of involved devices) and thus, offers statistics to the overall usage of an ATM network [10]. By providing various configuration options within our ATM-QOS-MIB, a very flexible network performance monitoring can be obtained this way.

The objective of the developed VCL-related QoS monitoring functions is to provide detailed diagnostics when one end point of a $\mathrm{VC}$ detects problems with the end-to-end QoS. In that case, the only way to identify the responsible device within the network requires measurements on each VCL of the connection and thus, on each ATM device involved. To that aim, we developed a set of monitoring functions [12] which are based on the VC tracing described in the previous section. The requesting user only has to specify one end point of the VC and the QoS module automatically starts to monitor the requested parameters on each link. The resulting measurements are then provided in a special data table of the ATM-QOS-MIB.

\section{PVC Manager}

Management communication within FuMA is generally based on SNMP. Thus, our primary goal in designing the PVC Manager was to implement a FuMA management application providing a Web-based interface to management middleware and element management, but requiring no knowledge of SNMP itself [9 12].

Focusing on permanent virtual connections (PVCs), the PVC Manager integrates a comprehensive amount of functionality concerning the areas configuration, performance, and fault management. Moreover, the implementation as Java applet guarantees the flexibility and extensibility necessary in today's heterogeneous networks.

As shown in Fig. 3, the architecture of the PVC Manager is basically composed of four distinct modules: topology discovery and display, connection management, device information, and QoS monitoring. While the first two modules are explained more detailed in the following sections, the functionality of the latter two is given very shortly: Basic management information about single ATM devices (e.g. the ifTable of the MIBII) is provided by the device information module, whereas the QoS monitoring module is responsible for triggering the monitoring process in the ATM-QOS-MIB as well as for acquiring and displaying measurements periodically [10].

\subsection{Topology Discovery and Display}

The module topology discovery and display is essential for the graphical user interface of the application. After launching the applet, the ANEMON is contacted and used to 


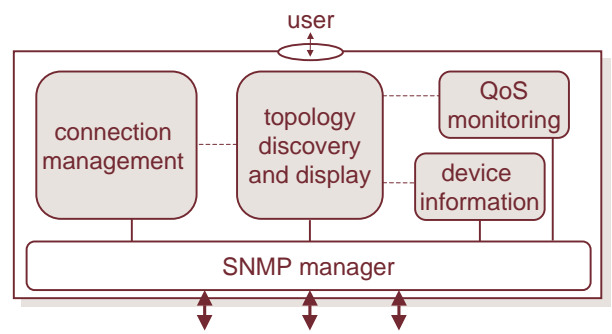

Fig. 3. Modules of the PVC Manager

generate the graphical representation of the network topology by reading the ATMTOPOLOGY-MIB (see Sect. 3.1). Each device is classified as end system, VC/VP switch, or VP switch (recognizable by the usage of different icons) while the capacity of single physical links is outlined by the thickness of the corresponding line (see Fig. 4). Concentrating on management functionality itself, no algorithm for the 'perfect' placement of all topology elements was included. Instead, the basic layout can be modified by the user (the graphical representation shown in Fig. 4 is the result of manual adjustments as well). Finally, as all modules rely on the process of topology discovery, the parameters of the topology module of the ANEMON are fully configurable, for example, starting point of the search, update interval, and search depth.

\subsection{Connection Management}

The module connection management implements the interface to the VC related functions of the element management as well as the access to the VC management module of the ANEMON. Because of its complexity, the module itself was split into three different sub-modules VC configuration, VC handling, and VC information.

Initially, $\mathrm{VC}$ configuration provides an efficient interface to the atmVcl/VplTable

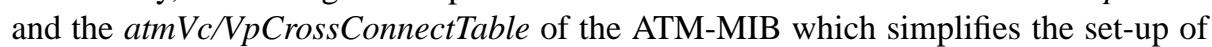
virtual links and cross-connects by the usage of graphic dialogues instead of inconvenient SNMP operations. In addition to useful filtering capabilities (e.g. only existing, non-terminating VCLs are candidates for cross-connecting), row-creation, activation, and configuration of traffic parameters in the atmTrafficDescrParamTable are not visible and performed as one single step. Furthermore, by relying on the ATM-VCC-MIB of the ANEMON, the PVC Manager allows the set-up of complete permanent virtual connections. This includes the selection of source and destination (or the whole transmission path) of a connection by simple mouse-clicks as well as the configuration of all other significant parameters (see Fig. 5). However, the procedure of actual VC set-up is entirely left to the ANEMON by using the ATM-VCC-MIB (see Sect. 3.2).

Supplementary functions are located in sub-modules two and three, VC handling and VC information, which primarily are responsible for provisioning functions concerned with the deletion of virtual links, cross-connects, and connections along with the display of important managed objects of the ATM-MIB. Another feature included 


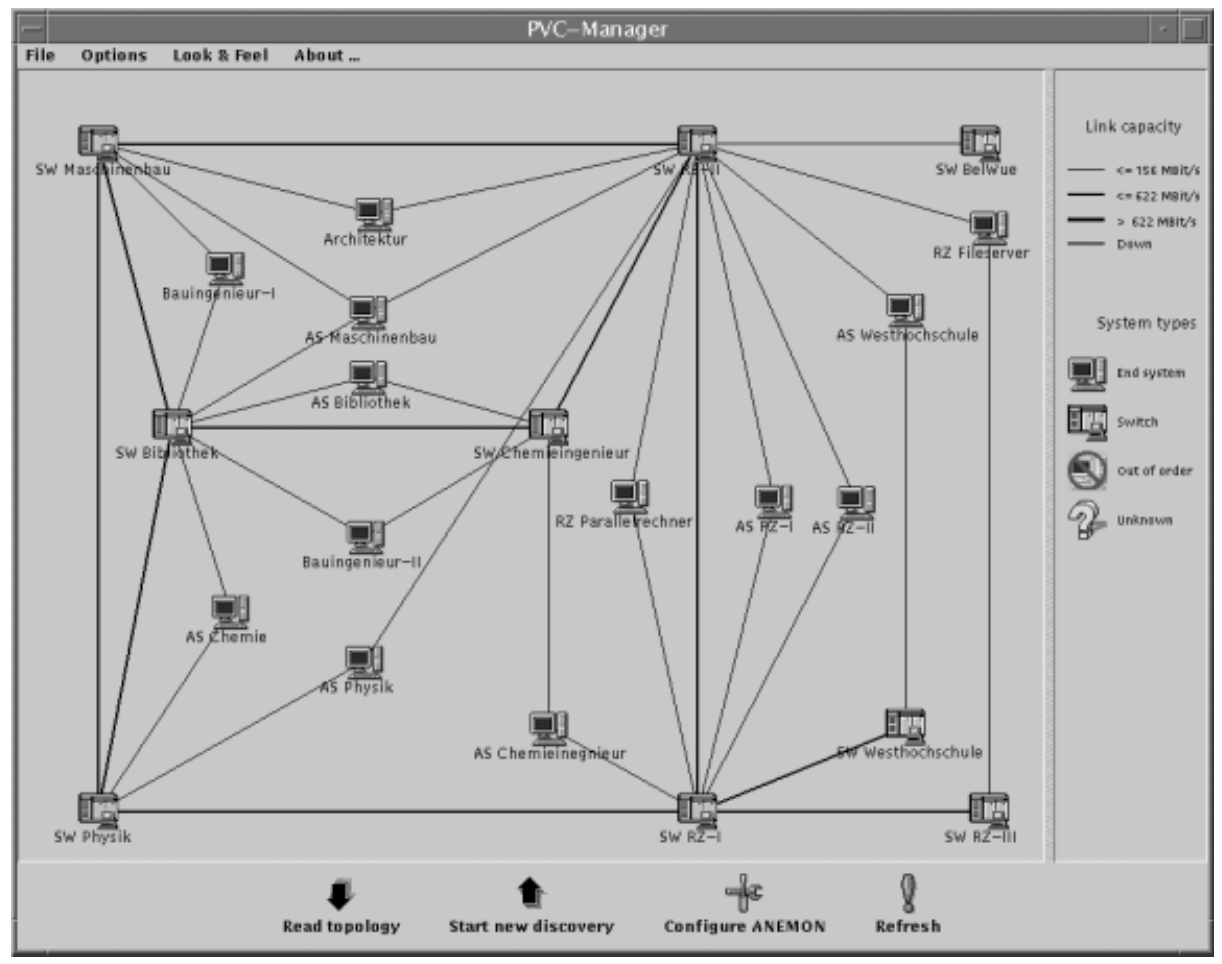

Fig. 4. Result of Topology Discovery

is the tracing of existing connections, which is based on the corresponding function of the ATM-VCC-MIB (see Sect. 3.2).

\section{Evaluation in a Simulated Environment}

In most of the tests performed, we used a simulation environment which has been specifically designed for evaluating ATM management functions [11]. The whole simulation environment is based on so-called virtual ATM devices consisting of a software management agent which operates on internal data only and thus, is able to simulate the behavior of a physical ATM device in a realistic way. The main advantage of this simulation approach compared with a hardware testbed is its scalability because small software agents each simulating one physical device can be placed in almost any amount within a simulation set-up. Another aspect is the very short time necessary to construct and simulate different scenarios.

The simulation scenario chosen for this presentation is based on a network topology which has been derived from the topology of an existing network, the ATM backbone of the University of Karlsruhe [11,12]. Focusing on ATM devices only, this backbone network consists of 9 switches and 13 end systems connected by physical links which 


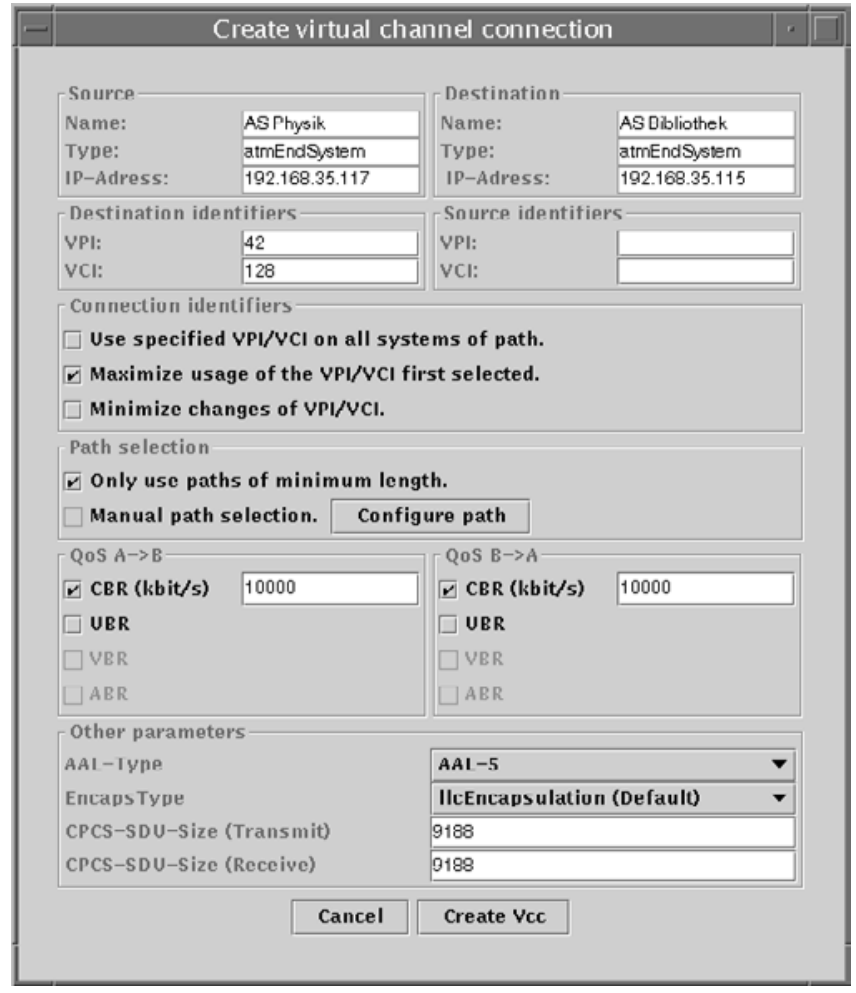

Fig. 5. Dialogue for the Set-Up of a VCC

run at $622 \mathrm{Mbps}$ between switches and $155 \mathrm{Mbps}$ between switches and end systems (see Fig. 4). On top of that scenario, we tested the ANEMON together with the PVC Manager as described in the following two sections.

\subsection{Testing Topology Discovery}

In this test, the PVC Manager was used in order to configure the topology discovery of the ANEMON (e.g. starting point), to initiate the discovery process, and to display the resulting topology. Fig. 4 shows the test result as provided by the ANEMON and displayed by the PVC Manager (after some manual re-arrangement, see Sect. 4.1 when all devices in the scenario are operational.

Our performance measurements [11/12] show that all 22 devices and 38 links of the topology are discovered correctly in approximately 4 seconds, even if some of the physical links are not in operational state (see Table 1). Though the effect of a failure of a whole device depends on several factors, obviously all devices within the topology are completely recognized only if there is at least one "bypassing" link, i.e. if the network does not fall apart into isolated sections by the removal of the defective device and its 
Table 1. Performance of the Topology Discovery

\begin{tabular}{|l|l|l|l|}
\hline Discovery Scenario & Devices & Links & Time Span \\
\hline Operational network & 22 & 38 & 4 sec. \\
\hline Broken link & 22 & 38 & 4 sec. \\
\hline End system failure & 22 & 38 & 38 sec. \\
\hline Switch failure & 21 & 37 & 38 sec. \\
\hline
\end{tabular}

adjacent links. As shown in Table 1 the simulated failure of one switch leads to the loss of one device and one link in the discovered topology because the missing device is only connected to the defective switch, whereas the simulated failure of one end system leads to a complete discovery. In both cases, the time span of 38 seconds is mainly determined by the extensive retry and timeout mechanisms of the ANEMON.

Over all, the tests proved the functional correctness and a good performance for the topology discovery. For instance, the performance enables up to fifteen discovery cycles a minute for a medium sized ATM network which is absolutely sufficient for rather static topology information.

\subsection{Testing PVC Management}

The tests presented in this section focus on the network-wide PVC management functionality, especially the PVC set-up process. Using the graphical interface of the PVC Manager, the appropriate inputs are made and then, the set-up process is triggered at the ANEMON. If the set-up is successfully completed, the PVC Manager displays a new window (see Fig. 6) containing the resulting VC data provided by the ANEMON. Moreover, the path of the new VC is highlighted in the topology view (see Fig. 4).

Table 2. Results of PVC Set-Up Tests

\begin{tabular}{|l|l|l|l|}
\hline No. Function & Description & Time Span \\
\hline 1. & $\begin{array}{l}\text { Creation of a } \\
\text { VCC }\end{array}$ & $\begin{array}{l}\text { QoS set to CBR (10 Mbps) for both } \\
\text { directions, } 4 \text { hops (3 physical links), } \\
\text { pre-configured transmission path and VPI/VCI }\end{array}$ & 8 sec. \\
\hline 2. & $\begin{array}{l}\text { Creation of a } \\
\text { VCC }\end{array}$ & $\begin{array}{l}\text { Same configuration as 1. but } \\
\text { with automatic path calculation }\end{array}$ & $8 \mathrm{sec.}$ \\
\hline 3. & $\begin{array}{l}\text { Creation of a } \\
\text { VCC }\end{array}$ & $\begin{array}{l}\text { Same configuration as 2. but } \\
\text { automatic VPI/VCI selection }\end{array}$ & 9 sec. \\
\hline
\end{tabular}

As Table 2 shows, the whole set-up process takes only 8 seconds in this test. Additionally, another test turned out that despite the simulated network is of considerable size, the time needed for path calculation can be neglected since the test took 8 seconds 


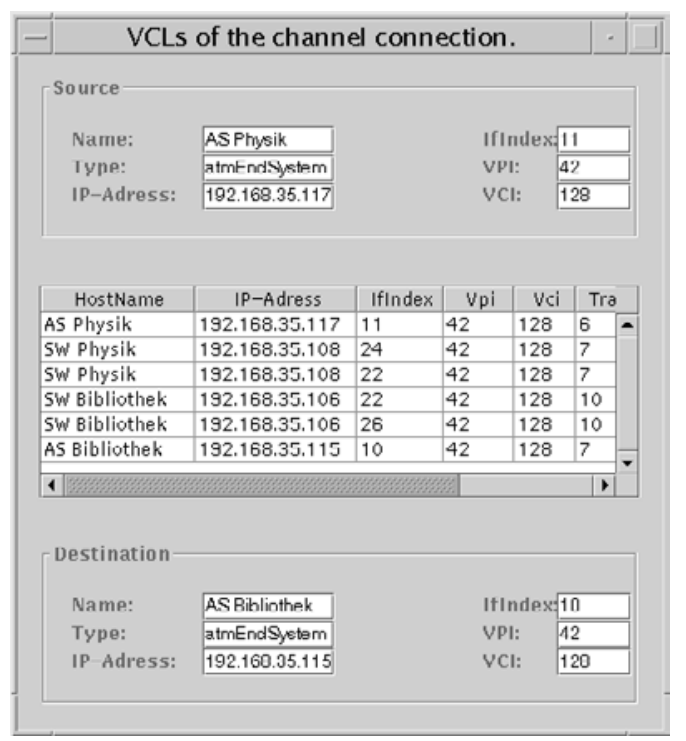

Fig. 6. Result of PVC Set-Up

for a pre-configured path as well. However, if the connection identifiers are chosen automatically (taking about $9 \mathrm{sec}$.) the whole set-up process is highly depending on how many VCs (i.e. VPIs and VCIs) are already in use.

To summarize, the PVC set-up tests proved the functionality and performance of our developed management entities. A PVC set-up time of about 8 seconds is a very good result for our medium sized network environment considering the amount of SNMP operations that have to be carried out in order to enter the desired values in the local MIBs distributed over all involved devices.

\section{Conclusion and Future Work}

In this paper, we presented our approach for ATM network management which mainly focuses on the important network-wide management functions required for ATM. The framework of our approach is given by the developed Function-oriented Management architecture for ATM networks (FuMA) which classifies management functions according to their functionality and view. Two of the components developed for our framework have been described in detail: Firstly, the ATM Network Monitor (ANEMON) which provides management functions for the important areas topology discovery, VC management, and QoS monitoring, and secondly, the PVC Manager which implements a graphical user interface to all functions of the ANEMON as well as to a lot of enhanced management functions of individual ATM devices. In the performed tests, both presented management components proved their functionality and showed a good performance using a specially designed simulation environment. To summarize, our approach 
provides a framework and some of the most essential functions for network-wide ATM management. Focusing on standardized and commonly accepted management interfaces only, the FuMA approach can be seen as a first important step towards global ATM management which is able to operate in a heterogeneous environment including ATM devices from different vendors. Moreover, the presented concept of the FuMA architecture, especially the management middleware, is generally applicable to management in distributed environments when global or network-wide management functions are required.

Future work primarily is concentrated on applying the developed and successfully tested management entities to a physical network. To that aim, we will cooperate closely with the University Computing Center of Karlsruhe in order to deploy our solutions in the real ATM backbone of the university. Currently, our main activities focus on adapting our topology discovery to the proprietary management interface of the ATM devices in that network and afterwards, continuously obtaining statistical data on the daily traffic by using the developed monitoring functions [10]. Besides practical tests, future work also concerns the development of additional or extended functions for our two entities as well as some more management entities for our FuMA framework.

\section{References}

1. The ATM-Forum. Integrated Local Management Interface (ILMI) Specification Version 4.0. af-ilmi-0065.000, September 1996.

2. E. W. Dijkstra. A Discipline of Programming. Prentice-Hall, 1976.

3. Object Management Group. Common Object Request Broker Architecture. Rev. 2.0, July 1995.

4. A. A. Lazar, S. K. Bhonsle, and K.-S. Lim. A Binding Architecture for Multimedia Networks. Journal of Parallel and Distributed Computing, 30(2):204-216, November 1995.

5. K. McCloghrie and M. Rose. Management Information Base for Network Management of TCP/IP-based internets: MIB-II. RFC 1213, March 1991.

6. Newbridge. MainStreetXpress Network Management Release 2.0. General Information Book, 1998.

7. H. Ritter, F. Fock, and H. R. Wiltfang. A Flexible Management Gateway for ATM-based Networks. Internal Report No. 20/98, University of Karlsruhe, Department of Computer Science, September 1998.

8. K. Tesink. Definitions of Managed Objects for ATM Management. RFC 2515, February 1999.

9. B. Thurm. Web-based Management of ATM Connections. Master's thesis, Institute of Telematics, University of Karlsruhe, Germany, February 1999.

10. B. Thurm and H. R. Wiltfang. Link-based Performance Monitoring of ATM Networks. In Proceedings of the 25th Conference of Local Computer Networks (LCN), Tampa, Florida, USA, 8. - 10. November 2000.

11. B. Thurm and H. R. Wiltfang. Simulating ATM Network Management using Virtual Devices. In Proceedings of the 2000 IEEE / IFIP Network Operations and Management Symposium, Honolulu, Hawaii, USA, 10. - 14. April 2000.

12. H. R. Wiltfang. Function-oriented Management of heterogeneous ATM Networks. PhD thesis, Institute of Telematics, University of Karlsruhe, Germany, February 1999.

13. H. R. Wiltfang and F. Fock. Topology Discovery for ATM Networks. In Proceedings of the 9th IEEE Workshop on Local and Metropolitan Area Networks, Banff, Canada, 17. - 20. May 1998. 